\title{
SÍNDROME MÃO-PÉ INDUZIDA POR CAPECITABINA: RELATO DE CASO
}

\author{
Walfrido Bispo Júnior ${ }^{1}$, Anna Cláudia de Andrade Tomaz², Larissa Fernanda de Araújo Vieira³, Patrícia \\ Magalhães Xavier Silva ${ }^{4}$, Silvana Maria Barros Oliveira ${ }^{5}$, Monik Kelly Santos Lima ${ }^{6}$
}

\begin{abstract}
RESUMO: A capecitabina é um agente quimioterápico indicado, dentre outros casos, como monoterapia no câncer de mama metastático. A síndrome mão-pé consiste numa das reações adversas associadas ao seu uso e caracteriza-se por eritema doloroso, edema, disestesia, descamação, bolhas e úlceras nas regiões palmar e plantar. Objetivou-se descrever o caso de uma paciente portadora de carcinoma de mama esquerda com metástase hepática que apresentou síndrome mão-pé decorrente do tratamento com capecitabina. Trata-se de um relato de caso ocorrido num hospital universitário de um estado do nordeste do Brasil em 2016. Foi necessária a suspensão do protocolo antineoplásico utilizado. Observou-se remissão parcial dos sintomas após o uso do gel de Aloe vera para o tratamento das áreas afetadas. Ressalta-se a importância do relato para conhecimento dessa reação adversa, facilitando sua identificação e manejo, para promover melhora da qualidade de vida do paciente oncológico.
\end{abstract}

DESCRITORES: Síndrome mão-pé; Antineoplásicos; Neoplasias da mama.

\section{CAPECITABINE-INDUCED HAND-FOOT SYNDROME: A CASE REPORT}

ABSTRACT: Capecitabine is a chemotherapeutic agent indicated, among other things, as monotherapy for metastatic breast cancer. Hand-foot syndrome is one of the adverse effects associated with its use and is characterized by painful erythema, edema, dysesthesia, desquamation, blistering and ulcers in the palms and soles. This study presents a case report on a patient with left-sided breast cancer metastasized to the liver, who suffered from capecitabine-induced hand-foot syndrome. The patient was being treated at a university hospital in a state in the Northeast of Brazil in 2016. Her antineoplastic protocol had to be suspended. After the use of Aloe vera gel to treat the affected areas, there was a partial remission of symptoms.It is important to expand knowledge about this adverse reaction, facilitating its identification and management, in order to improve quality of life in cancer patients.

DESCRIPTORS: Hand-foot Syndrome; Antineoplastic Agents; Breast Neoplasms.

\section{SÍNDROME MANO-PIE INDUCIDO POR CAPECITABINA: RELATO DE CASO}

RESUMEN: La capecitabina es un agente quimioterápico indicado, entre otros casos, como monoterapia en cáncer de mama metastático. El síndrome mano-pie consiste en una de las reacciones adversas asociadas a su uso, caracterizada por eritema doloroso, edema, parestesia, descamación, ampollas y úlceras en regiones palmar y plantar. Se objetivó describir el caso de una paciente con carcinoma en mama izquierda, con metástasis hepática presentando síndrome de mano-pie derivado del tratamiento con capecitabina. Relato de caso ocurrido en hospital universitario de estado del Noreste brasileño en 2016. Fue necesaria la suspensión del protocolo antineoplásico utilizado. Se observó remisión parcial de los síntomas luego de uso de gel de Aloe Vera para tratamiento de las áreas afectadas. Se destaca la importancia del relato para conocimiento de esta reacción adversa, permitiendo su identificación y manejo, promoviendo mejora de calidad de vida del paciente oncológico.

DESCRIPTORES: Síndrome Mano-Pie; Antineoplásicos; Neoplasias de la Mama.

${ }^{1}$ Farmacêutico. Doutor em Biotecnologia Aplicada à Saúde. Farmacêutico do Hospital Universitário Professor Alberto Antunes. Maceió, AL, Brasil.

${ }^{2}$ Farmacêutica. Doutora em Produtos Naturais. Farmacêutica do Hospital Universitário Professor Alberto Antunes. Maceió, $\mathrm{AL}$, Brasil.

${ }^{3}$ Farmacêutica. Mestre em Ciências da Saúde. Farmacêutica do Hospital Universitário Professor Alberto Antunes. Maceió, AL, Brasil.

${ }^{4}$ Farmacêutica. Especialista em Farmacologia Clínica. Farmacêutica do Hospital Universitário Professor Alberto Antunes. Maceió, AL, Brasil.

${ }^{5}$ Enfermeira. Mestre em Enfermagem. Enfermeira do Hospital Universitário Professor Alberto Antunes. Maceió, AL, Brasil.

${ }^{6}$ Enfermeira. Especialista em Oncologia. Enfermeira do Hospital Universitário Professor Alberto Antunes. Maceió, AL, Brasil.

Autor Correspondente:

Recebido: $10 / 03 / 2016$

Walfrido Bispo Júnior

Hospital Universitário Professor Alberto Antunes

Finalizado: 20/12/2016

Av. Lourival Melo Mota, s/n - 57072-900 - Maceió, AL, Brasil

E-mail: walfrido.bispo@hu.ufal.br 


\section{INTRODUÇÃO}

A capecitabina é uma fluoropirimidina, pró-droga sistêmica do 5-fluorouracil (5-FU), com a vantagem da administração oral. É indicada para uso na terapia adjuvante do tratamento do câncer colorretal, terapia de primeira linha em câncer colorretal metastático e como monoterapia ou em combinação com docetaxel no câncer de mama metastático. As toxicidades dose-dependentes mais comuns associadas à capecitabina são hiperbilirrubinemia, diarreia e síndrome mão-pé (SMP) ${ }^{(1)}$.

Os agentes quimioterápicos comumente causadores dessa síndrome, além da capecitabina, são: doxorrubicina lipossomal peguilada, 5-fluorouracil, citarabina e docetaxel. Novos inibidores multiquinase como sorafenibe, sunitinibe, axitinibe, pazopanibe, regorafenibe e vemurafenibe também podem ocasionar a SMP(2).

A SMP, também conhecida como eritrodisestesia palmo-plantar ou eritema acral, é uma reação adversa decorrente da terapia com agentes quimioterápicos e biológicos ${ }^{(3)}$ que foi primeiramente descrita em associação com o mitotano por Zuehlke em 1974(2). Nos casos leves a moderados, podem ocorrer eritema doloroso e edema, vários graus de disestesia, que podem ser seguidos por descamação úmida ou seca das palmas das mãos e solas dos pés. Já em casos mais graves ocorrem: rachaduras, descamações da pele, bolhas, úlceras e dores intensas que podem interferir nas atividades diárias ${ }^{(3)}$.

Tendo em vista que a SMP traz importante impacto na qualidade de vida do paciente oncológico, faz-se necessária a documentação de casos dessa reação adversa no sentido de contribuir para a ampliação do conhecimento e facilitar sua identificação e manejo. Desta feita, objetivou-se relatar um caso de ocorrência de SMP numa paciente de um hospital universitário de um estado do nordeste do Brasil.

\section{RELATO DE CASO}

Trata-se da paciente D.S.S., do sexo feminino, 39 anos, parda, professora, portadora de carcinoma de mama esquerda com metástase hepática, tratada inicialmente com quimioterapia neoadjuvante com doxorrubicina e ciclofosfamida, seguida de docetaxel (AC-T) por um ciclo, a qual foi substituída pelo tratamento com paclitaxel semanal e trastuzumabe.

O paclitaxel foi suspenso devido ao desenvolvimento de neurotoxicidade grau II limitante, sendo iniciado o uso de capecitabina na dose de 3,5g/dia por 14 dias, em ciclos de 21 dias. Durante o $3^{\circ}$ ciclo, a paciente relatou dor, eritema, edema, formigamento e descamação nas regiões palmar e plantar, especialmente desta última, sendo indicativos da síndrome mão-pé relacionados ao uso de capecitabina. A conduta médica foi a suspensão da capecitabina e início de tratamento com docetaxel.

Após o $15^{\circ}$ dia, a paciente retornou ao hospital apresentando evolução do quadro álgico, edema, descamações, presença de bolhas e limitação para deambular, com dificuldade para realizar as atividades do cotidiano, sendo diagnosticada com SMP grau II, em concordância com a classificação proposta pelo National Cancer Institute ${ }^{(4)}$.

Posteriormente, após avaliação da enfermagem, a paciente iniciou o uso de gel de Aloe vera nas áreas lesionadas, três vezes ao dia. Houve remissão parcial dos sintomas após duas semanas de uso, conforme observado na visita realizada pela farmacêutica clínica. Este caso foi acompanhado por, aproximadamente, dois meses. A partir da identificação da SMP e manejo terapêutico exitoso, observou-se significativa melhoria da qualidade de vida da paciente.

\section{DISCUSSÃO}

O diagnóstico da SMP é baseado em suas características clínicas ${ }^{(5)}$ e, neste relato de caso, a paciente foi diagnosticada como grau II. A SMP grau I envolve mudanças mínimas na pele ou dermatite (como eritema, edema ou hiperqueratose) sem dor, enquanto a SMP grau III caracteriza-se por mudanças severas na pele (como descamação, bolhas, sangramento, edema ou hiperqueratose) com dor e 
limitações no auto-cuidado ${ }^{(4)}$.

Dentre os medicamentos utilizados durante a terapia antineoplásica da paciente, além da

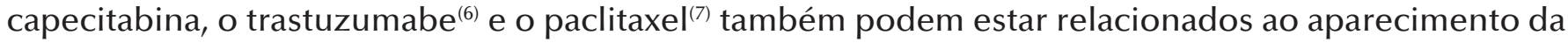
SMP. No entanto, foi observado que o surgimento desta síndrome ocorreu após o uso da capecitabina e houve remissão dos sintomas mediante a suspensão do tratamento com a mesma.

Em geral, a SMP ocorre nas semanas iniciais da terapia com o agente desencadeante; no entanto, para alguns medicamentos como a capecitabina, a mesma pode ocorrer em até alguns meses após o início do tratamento ${ }^{(8)}$, condizente com a situação descrita. Testes clínicos chaves sobre o uso de diferentes doses de capecitabina no tratamento de câncer de mama indicam incidência de 32-62\% para todos os graus de $\mathrm{SMP}^{(9)}$.

A dose utilizada do agente quimioterápico bem como o gênero feminino são fatores de risco para a $\mathrm{SMP}^{(2)}$. A presença de hiperqueratose, eczema, doença fúngica e mau alinhamento que resulte em distribuição desigual da pressão são fatores predisponentes que também devem ser avaliados antes do início da terapia e tratados em caso de necessidade ${ }^{(8)}$.

A opção utilizada como tratamento tópico das lesões apresentadas pela paciente foi o gel a base de Aloe vera. Este possui propriedades umectantes, emolientes, anti-inflamatórias, cicatrizantes e regeneradoras de tecidos ${ }^{(10)}$.

Foi observada a remissão parcial dos sintomas da paciente, fato que se assemelha a um relato de caso $^{(10)}$ que também evidenciou melhora significativa do quadro de SMP, induzida por quimioterapia, ao utilizar gel aquoso de Aloe vera, com base em suas propriedades e em referências do uso para dermatites decorrentes de radioterapia. No relato os autores discorrem que não há outros estudos sobre o uso desse fitoterápico no tratamento da SMP(10). Uma pesquisa mostrou o uso de produtos para cuidados da pele que possuíam Aloe em sua composição para o alívio sintomático da SMP(11).

O manejo mais efetivo da SMP consiste no aumento do intervalo entre as administrações do fármaco capecitabina, diminuição da dose ou interrupção do tratamento ${ }^{(12)}$, em concordância com a conduta médica realizada. Outras medidas para o tratamento da SMP incluem o uso de emolientes tópicos, antibióticos para prevenção de infecções secundárias, esteroides tópicos, piridoxina e inibidores da ciclooxigenase $2^{(1)}$.

\section{- CONCLUSÃo}

Tendo em vista que a capecitabina é um agente quimioterápico comumente utilizado e está relacionada ao aparecimento da SMP, é de suma importância o seu conhecimento e diagnóstico clínico. A equipe multidisciplinar deve estar atenta para que medidas preventivas e de acompanhamento do tratamento sejam realizadas, com o intuito de melhorar a qualidade de vida do paciente. Diante do resultado positivo obtido com a utilização do gel de Aloe vera no tratamento sintomático da SMP, sugere-se que estudos adicionais sejam realizados a fim de elucidar a repercussão acerca não apenas do seu uso, mas também como medida profilática para esta condição.

\section{- REFERÊNCIAS}

1. Sanghi S, Grewal RS, Vasudevan B, Nagure A. Capecitabine induced hand-foot syndrome: report of two cases. Armed Forces Med J India. [Internet] 2013;69(1) [acesso em 16 fev 2016]. Disponível: http://dx.doi.org/10.1016/j. mjafi.2012.01.009.

2. Miller KK, Gorcey L, McLellan BN. Chemotherapy-induced hand-foot syndrome and nail changes: A review of clinical presentation, etiology, pathogenesis, and management. J Am Acad Dermatol. [Internet] 2014;71(4) [acesso em 16 fev 2016]. Disponível: http://dx.doi.org/10.1016/j.jaad.2014.03.019.

3. Surjushe A, Vasani R, Medhekar S, Thakre M, Saple DG. Hand-Foot syndrome due to capecitabine. Indian J Dermatol. [Internet] 2008;53(1) [acesso em 16 fev 2016]. Disponível: http://dx.doi.org/10.4103/0019-5154.39747. 
4. National Cancer Institute (NCI). Common Terminology Criteria for Adverse Events (CTCAE). Version 4.03. [Internet] 2010;4(3) [acesso em 07 mar 2016]. Disponível: http://evs.nci.nih.gov/ftp1/CTCAE/ CTCAE_4.03_2010-06-14_QuickReference_5x7.pdf.

5. Qiao J, Fang H. Hand-foot syndrome related to chemotherapy. CMAJ. [Internet] 2012;184(15) [acesso em 16 fev 2016]. Disponível: http://dx.doi.org/10.1503/cmaj.111309.

6. Tho LM, Bose N, Robertson L, Bhattacharya S, McClue L, Anderson L, et al. Trastuzumab-related palmar plantar erythrodysaesthesia. J Clin Oncol. [Internet] 2012;24(1) [acesso em 08 mar 2016]. Disponível: http://dx.doi. org/10.1016/j.clon.2011.10.002.

7. Richards KN, Ivan D, Rashid RM, Chon SY. Paclitaxel-induced acral erythema. Arch Dermatol. [Internet] 2012;148(11) [acesso em 08 mar 2016]. Disponível: http://dx.doi.org/10.1001/archdermatol.2012.2830.

8. Degen A, Alter M, Schenck F, Satzger I, Völker B, Kapp A, et al. The hand-foot-syndrome associated with medical tumor therapy - classification and management. J Dtsch Dermatol Ges. [Internet] 2010;8(9) [acesso em 16 fev 2016]. Disponível: http://dx.doi.org/10.1111/j.1610-0387.2010.07449.x.

9. Gresset SM, Stanford BL, Hardwicke F. Management of hand-foot syndrome induced by capecitabine. J Oncol Pharm Pract. [Internet] 2006;12(3) [acesso em 08 mar 2016]. Disponível: http://dx.doi.org/10.1177/1078155206069242.

10. Simão DAS, Lima EDRP, de Souza RS, Faria TV, Azevedo GF. Síndrome mão-pé induzida por quimioterapia: relato de um caso. Rev. bras. enferm. [Internet] 2012;65(2) [acesso em 16 fev 2016]. Disponível: http://dx.doi. org/10.1590/S0034-71672012000200026.

11. Schwandt A, Wood L S, Rini B, Dreicer R. Management of side effects associated with sunitinib therapy for patients with renal cell carcinoma. Onco Targets Ther. [Internet] 2009;(2) [acesso em 08 ago 2016]. Disponível: https://www.ncbi.nlm.nih.gov/pmc/articles/PMC2886329/.

12. Farr KP, Safwat A. Palmar-plantar erythrodysesthesia associated with chemotherapy and its treatment. Case Rep Oncol. [Internet] 2011;4(1) [acesso em 16 fev 2016]. Disponível: http://dx.doi.org/10.1159/000327767. 